\title{
Electron dynamics method using a locally projected group diabatic Fock matrix for molecules and aggregates
}

\author{
Takehiro Yonehard* and Takahito Nakajima \\ RIKEN Center for Computational Science, Kobe 650-0047, Japan
}

(Dated: August 23, 2019)

\begin{abstract}
We propose a method using reduced size of Hilbert space to describe an electron dynamics in molecule and aggregate based on our previous theoretical scheme [ T. Yonehara and T. Nakajima, J. Chem. Phys. 147, 074110 (2017) ]. The real-time time-dependent density functional theory is combined with newly introduced projected group diabatic Fock matrix. First, this projection method is applied to a test donor-acceptor dimer, namely, a naphthalene-tetracyanoethylene with and without initial local excitations and light fields. Secondly, we calculate an absorption spectrum of five-unit-polythiophene monomer. The importance of feedback of instantaneous density to Fock matrix is also clarified. In all cases, half of the orbitals were safely reduced without loss of accuracy in descriptions of properties. The present scheme provides one possible way to investigate and analyze a complex excited electron dynamics in molecular aggregates within a moderate computational cost.
\end{abstract}

\section{INTRODUCTION}

Recently, the authors proposed a concise method for describing the quantum dynamics of excited electrons traveling over constituent molecules in a molecular aggregate system by utilizing a group diabatic Fock (GDF) matrix ${ }^{1}$. The construction scheme for this GDF matrix using atom centered Gaussian basis set was practically demonstrated by Thoss et al $\underline{\underline{2}}$ based on the idea of diabatization of block diagonalization of Hamitonian that was originally introduced by Köppel et al $\underline{\underline{3}}$ Before that, the applications of this block diabatization concept for a practical calculation can be found in the works on electron conduction problems. $\frac{4,5}{, 5}$

This block diabatic scheme for the electron dynamics provides an intuitive understanding of charge and exciton migration as the quantum mechanical transport of electronic energies in a molecular assembly subject to the inherent electronic propensities of the site molecules, starting from any prepared type of initial local excitations considering the light-electron couplings.

In the present article, on the basis of the characteristics of a nearly block structured diabatic representation in the GDF method, we introduce a compact representation within an extracted Hilbert subspace. The aim of this paper is two-fold: (1) to introduce a scheme for reducing the computational cost in keeping with an accurate description of the dynamics, and (2) to investigate how many local orbitals are required for a description of dynamics and absorption spectrum toward an interpretation of electron dynamics.

Note that the group diabatic representation treated here does not lead by itself to a reduction of computational cost by describing the dynamics with a small subset of the full orbital space. In fact, the GDF matrix has the same dimension as the original Fock matrix before the block diagonalization. (1) and (2) are obtained by using a projection scheme introduced here as an extraction of local group diabatic orbitals associated with a size of the Hilbert space needed for a description of electron dynamics. The reduction of calculation cost is associated with the reduction of the number of extracted local group orbitals as a basis set for constructing matrix representations of electronic operators utilized in a calculation of electron dynamics.

This article improves upon our previous work by providing and assessing a procedure for reducing the computational cost of electron dynamics calculation using the GDF method, which is combined with the real-time timedependent density functional theory (RT-TDDFT) ${ }^{\underline{6}}-\underline{\underline{9}}$ under the adiabatic approximation for electron functionals.

The point is that electron migration of chemical interest over a molecular aggregate under moderate light conditions occurs in a sub Hilbert space consisting of a low number of excited states described by molecular orbitals within a relevant but not very large energy range around the Fermi energy of a system.

It is instructive to compare other studies with our present method. There are many studies intensively investigating molecular interactions and their effects on excited energy transfer proceeding in excited molecular aggregates $\stackrel{10-22}{\underline{2}}$ For example, Futera et al $\underline{\underline{23}}$ successfully utilized and assessed the GDF method originally named a projection operator diabatization scheme $e^{2}$ to evaluate electronic coupling matrix elements with high level ab initio calculations in the electron-transfer process of a molecular-metal/semiconducter interface. However, a study on a systematic variable description of the excited electron dynamics in a bottom-up approach is rare. Compared to previous studies on excited electron transfer in molecular aggregates, the scheme introduced in the present article has the advantages of a compact description of excited electron migration with ab initio electronic structure calculations. In addition our new scheme allows a systematic improvement of the results by enlarging the projection space. The most prominent feature is that our scheme is intended for a real-time dynamics of excited electrons in molecular aggregates in an external field starting from any pattern of initial local excitations prepared as a perturbation for the electronic 
state.

In this article, we detail the procedures for constructing the projected local orbital space within the group diabatic (GD) representation, and then demonstrate numerical applications. We examine the size of a local orbital space related to dynamics in a systematic way by increasing an energy range for projecting a diabatic local orbital space. A naphthalene(NPTL) - tetracyanoethylene(TCNE) dimer is treated as a test donor-acceptor system. Additionally, we also demonstrate a convergence of absorption spectrum with respect to a size of orbital space using a five-unit polythiophene molecule(5UT) as a typical electron donor species in solar cell materials. To ensure the energy balance between local projected orbitals, we employ an energy width parameter for extracting a relevant subspace with the mean value of the highest occupied molecular orbital (HOMO) and lowest occupied molecular orbital (LUMO) energies in the whole system as a reference energy, and we do not use a scheme which requires direct orbital selection.

In Sect. II, we explain the theoretical method for describing the electron dynamics based on the GDF representation within projected local diabatic orbitals, which is followed by numerical examples in the section III.

\section{THEORETICAL METHOD}

In this section, after a brief summary of the electron dynamics method $1 \underline{1}$ using a GD representation, 1,10-13,17 we introduce a scheme for constructing a concise matrix form with use of projected diabatic local orbitals having a dominant contribution to the underlying dynamics. The determination of diabatic local projection orbitals playing a main role in the present work requires only the parameters of energy ranges for monomers covering the important orbitals around HOMO and LUMO playing in an excited electron dynamics.

\section{A. Overview of the locally projected-space group diabatic representation}

The transformation from an atomic orbital (AO) representation of physical operators to a GD form consists of the following two transformations being constructed sequentially:

(i) a transformation to a representation using the Löwdin orthogonalized atomic basis functions and

(ii) a unitary transformation made from the local orbital sets obtained by the diagonalizations of block sub-matrices corresponding to the predetermined monomer groups in the Fock matrix prepared in step (i).
The overview of the process of extracting the diabatic local projection orbitals as the primary topic of the present article is as follows:

(a) calculate the mean of the HOMO and LUMO orbital energies of the whole system;

(b) set an energy range covering the local orbitals for each monomer in which the value obtained in (a) is placed at the middle;

(c) obtain the diabatic local projection orbitals of the monomers in the energy range prepared in (b).

We refer to the matrix representation within these projection orbitals as the locally projected-space group diabatic Fock (LP-GDF) representation, of which the details are explained in later subsections.

The multiplications of matrices associated with the electronic properties and the analyses of the timedependent electron density using the newly introduced LP-GDF representation are carried out within the projected orbital space. The information related to the size of the orbital space required for a description of the dynamics without loss of accuracy provides us with insight into the sub-Hilbert space relevant to it.

In the following subsection, for a self-contained form of the present article, we first summarize the electron dynamics scheme using a GD representation ${ }^{1}$ and then proceed to describe how to obtain the diabatic local projection orbitals and how to construct compact matrix representations by using them as a subset of the basis functions.

\section{B. Group diabatic representation}

\section{Fock matrix in the Löwdin representation}

The first step is to prepare a Fock matrix represented by the Löwdin orthogonalized atomic basis function 24

$$
\widetilde{F}_{m n} \equiv\left\langle\widetilde{\chi}_{m}|\widehat{F}| \widetilde{\chi}_{n}\right\rangle
$$

where the orthogonalized Löwdin atomic orbitals(AOs) are expressed by

$$
\left|\widetilde{\chi}_{n}\right\rangle=\sum_{j}^{\mathrm{AO}}\left|\chi_{j}\right\rangle\left(\underline{\underline{S}}^{-1 / 2}\right)_{j n}
$$

with $S_{j n}=\left\langle\chi_{j} \mid \chi_{n}\right\rangle$ being the AO overlap matrix element. Here $\left\{\chi_{n}\right\}$ is the original basis set consisting of AOs.

\section{Localized orbitals of a subgroup}

After the classification of $\left\{\widetilde{\chi}_{n}\right\}$ into subgroups, e.g., monomers, the block structure of the Fock matrix within the Löwdin basis set is determined with its diagonal 
blocks $\left\{\underline{\underline{\widetilde{F}}}_{G_{i} G_{i}}\right\}$ and off-diagonal ones $\left\{\underline{\underline{\widetilde{F}}}_{G_{i} G_{j}}\right\}_{i \neq j}$ with $\mathrm{i}$ and $\mathrm{j}$ ranging from 1 to $N_{g}$. $G_{i}$ denotes the i-th subgroup. $N_{g}$ is the number of subgroups in the system. Note that we can employ arbitrary divisions of the component atoms in the whole system.

The diagonalization of diagonal blocks corresponding to subgroup $G$,

$$
\underline{\underline{F}}_{G G}=\underline{\underline{D}}_{G} \underline{\underline{F}}_{G G} \underline{\underline{D}}_{G}^{\dagger},
$$

gives rise to the unitary transformation matrix $\underline{\underline{D}}_{G}$, whose column vectors are the linear coefficient vectors of the localized eigenstates expanded in terms of the Löwdin orthogonalized atomic basis functions for the group $G$. The dagger symbol attached to a matrix indicates its adjoint form. $\underline{\underline{F}}_{G G}$ is the diagonal matrix having the eigen energies $\left\{\epsilon_{j, G}\right\}_{j=1 \sim M_{G}}$ of the corresponding subgroup $G$ as its elements, and $M_{G}$ is the number of local basis functions spanned at group $G$. Here, $G \in\left\{G_{1}, \ldots, G_{N_{g}}\right\}$.

The elements in off-diagonal blocks of the Fock matrix represented by these localized orbitals, associated with different groups, can take non-zero values, which provide a diabatic character in the representation with the use of the collection of these orbital sets. These group localized orbital sets provide a transformation matrix from a Löwdin representation to the GD one to be explained later.

Here, we provide a short comment on the ambiguity of group division in a Löwdin representation of Fock operator. In case using diffuse AO orbitals having far larger distribution than a distance between monomers, a center of Löwdin orbital created by the mixture of original AOs can become close to a nuclei belonging to a different group. Though that is one of the possible problem in the group assignment of Löwdin orbitals associated with a natural group sectoring, it does not cause any problem in case with distances of monomers sufficiently larger than AOs and also Löwdin orbitals $\frac{17}{1}$ In this article, we do not use diffuse orbitals and treat a case with distance of monomers being larger than a spatial range in orbital distribution.

\section{Group diabatic Fock matrix}

The GD representation of the Fock operator $\underline{\underline{F}}$, as one of the main ingredients in the GDF electron dynamics scheme is constructed via the transformation of the Löwdin representation matrix of the Fock operator $\widetilde{\underline{F}}$. By using the already obtained unitary matrices with the dimensions of the local basis functions associated with groups, $\left\{\underline{\underline{D}}_{G_{i}}\right\}_{i=1 \sim N_{g}}$, this transformation is expressed by $1,10-12$

$$
\underline{\underline{F}}_{G_{i} G_{j}}=\underline{\underline{D}}_{G_{i}}^{\dagger} \underline{\widetilde{F}}_{G_{i} G_{j}} \underline{\underline{D}}_{G_{j}},
$$

where $\mathrm{i}$ and $\mathrm{j}$ range from 1 to the number of groups $N_{\mathrm{g}}$. The assembly of these sub-matrices $\left\{\overline{\underline{F}}_{G_{i} G_{j}}\right\}_{i, j=1 \sim N_{g}}$ constructs the GD representation which is called GDF matrix and expressed by $\underline{\underline{F}}^{\mathrm{GD}}$. The physical meaning of the components in this final form is as follows. This sub-matrices $\left\{\underline{\underline{F}}_{G_{i} G_{i}}\right\}_{i=1 \sim N_{g}}$ in the diagonal blocks correspond to the local group eigen energies, while $\left\{\overline{\underline{F}}_{G_{i} G_{j}}\right\}_{i \neq j}$, placed at the off-diagonal blocks, describes the interactions between different groups. Note that, in this transformation, the information included in the $\mathrm{AO}$, Löwdin, and GDF representations are the same and no approximation is applied.

\section{Transformation from the AO representation to the GD representation}

A matrix representation of any observable operator $\hat{O}$ in terms of the constructed GD basis set, $\underline{O}^{\mathrm{GD}}$, is related to that of the original AO basis set, $\underline{\underline{O}}^{\mathrm{AO}}$, as

$$
\underline{\underline{O}}^{\mathrm{GD}}=\underline{\underline{U}}^{\dagger} \underline{\underline{O}}^{\mathrm{AO}} \underline{\underline{U}}
$$

where $\underline{\underline{U}} \equiv \underline{\underline{S}}^{-1 / 2} \underline{\underline{W}}$. Here, the diagonal block in the subtransformation matrix $\underline{\underline{W}}$ is given by $\underline{\underline{W}}_{G_{i} G_{i}} \equiv \underline{\underline{D}}_{G_{i}}$ for $i$, while the off-diagonal one is defined as $\underline{\underline{W}}_{G_{i} G_{j}} \equiv \underline{\underline{0}}$. The Fock matrix obeys the same transformation rule and is obtained by setting $\hat{O}=\hat{F}$ in the above equations, where we know that $\underline{\underline{F}}^{\mathrm{AO}}=\underline{\underline{F}}$ and $\underline{\underline{F}}^{\mathrm{GD}}=\overline{\underline{F}}$.

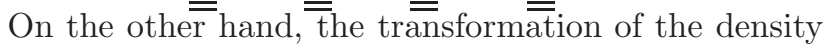
matrix from the original $\mathrm{AO}$ basis set to the GD one is written as

$$
\underline{\underline{\rho}}^{\mathrm{GD}}=\underline{\underline{U}}_{\underline{\underline{\rho}}}^{\mathrm{AO}} \underline{\underline{U}}^{\dagger}
$$

Note that the unitarity of $\underline{W}$ assures total electron conservation with respect to this transformation,

$$
\operatorname{Tr}\left[\underline{\underline{\rho}}^{\mathrm{AO}} \underline{\underline{S}}\right]=\operatorname{Tr}\left[\underline{\underline{\rho}}^{\mathrm{GD}}\right] .
$$

\section{State coupling}

We can obtain the essential elements needed for the construction of light-electron coupling by setting $\hat{O}=\hat{\mathbf{r}}$, $\partial_{\mathbf{r}}$ in the previous subsection. Here, boldface denotes a vector in a three-dimensional Cartesian space, and $\mathbf{r}$ denotes a composite variable of the electron position in three-dimensional space. The first and second operators are responsible for the light-electron coupling in length and velocity forms $\frac{25,26}{2}$ Here, we neglect the nonadiabatic coupling and molecular motion to allow us to focus on an examination of the electron dynamics scheme using projected local diabatic group orbitals.

Light-electron couplings within GD representation are expressed by $\underline{\underline{L}}^{\mathrm{GD}}=+e \underline{\underline{\mathbf{r}}}^{\mathrm{GD}} \mathbf{E}$ for the length gauge and

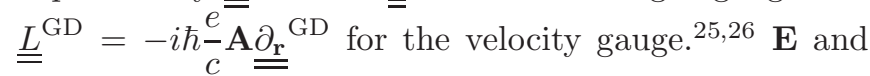


A denote respectively the three-dimensional electric field vector and electromagnetic field vector potential, which generally depend on a point in a three-dimensional space. $\underline{\underline{\mathbf{r}}}^{\mathrm{G} D}$ and ${\underline{\underline{\partial_{\mathbf{r}}}}}^{\mathrm{G} D}$ denote the GD presentation matrices respectively for $\hat{\mathbf{O}}=\mathbf{r}$ and $\partial_{\mathbf{r}}$ in Eq. (5). In this study, we employ the length gauge. The details on the treatment for these coupling matrices with further approximations applied in dynamics calculation are given in Subsect. IE

\section{Locally projected space made from subsets of local site orbitals}

This subsection is an essential part of the present article proposing the LP-GDF scheme. Here, we provide the concrete procedures used in it and the corresponding mathematical expressions.

Let us consider the diagonal block of the Fock matrix within the GD representation, $\left\{\underline{\underline{F}}_{G_{i} G_{i}}\right\}$ and the associated local orbital energies $\left\{\epsilon_{j, G_{i}}\right\}_{j=1 \sim N_{G_{i}}}$ corresponding to the $\mathrm{i}$-th group site. In a situation where the interactions between group monomers are weak compared to those among the atoms in each monomer, we can safely employ the referential local ground state, where all of the electrons assigned to a local site are filled in ascending order from the lowest-energy local orbital.

The excited electron dynamics in a molecular aggregate system under moderate sunlight conditions is expected to proceed in low-excited-state manifolds constructed from the local molecular orbitals in a relevant but not very large energy range around the Fermi energy of the system. In a situation involving rather strong light field or molecular interaction, the Hilbert subspace required for a description of the dynamics will become large. It is worthwhile to examine the size of the subspace relevant to the excited electron dynamics by varying the type of molecular interactions such as light-matter coupling and initial local excitations.

Thus, in order to examine the size of the orbital space needed for a description of the electron dynamics at a sufficient accuracy, we establish a procedure for projecting a Hilbert subspace around the Fermi energy of the whole molecular aggregate by using a width parameter for the energy range as follows:

(i) Set an energy width $\Delta \epsilon_{i}$ covering the sub-space consisting of local orbitals orbitals for the $i$-th monomer, $G_{i}$. Here $i$ ranges from 1 to $N_{g}$.

(ii) Calculate the mean $\eta$ of the HOMO and LUMO energies of the whole system.

(iii) Extract a subset of group diabatic local orbitals in the energy range $\left[\eta-\Delta \epsilon_{i} / 2, \eta+\Delta \epsilon_{i} / 2\right]$ from the whole orbital space. We refer to this as a projection of the diabatic local orbitals. (iv) Construct minor matrices as representations of the electronic operators within the projected orbital basis set obtained in (iii).

(v) Follow the same procedure as that in the GDF electron dynamics scheme other than the projection introduced here. This means that time propagation and property analysis are carried out using the obtained small size matrices.

The mathematical form of LP-GDF procedure is compactly given in Appendix C

In this article, we employ a version of projection independent on the orbitals during a time propagation but constructed from the initial orbitals in dynamics.

The orbital projection scheme using an energy width allows for the systematic and natural treatment for general situations involving the unknown energy orders and spatial localities of the GD orbitals in molecular aggregates.

Note that to ensure the energy balance between local projection orbitals, we employ an energy width parameter for extracting a relevant subspace with the mean value of the highest occupied molecular orbital (HOMO) and lowest occupied molecular orbital (LUMO) energies in the whole system as a reference energy, and we do not use a scheme which requires direct orbital selection.

In the following numerical demonstrations, for focusing on this essential point in the proposed scheme, we employ a parameter for the energy range independent of the monomers, namely, $\Delta \epsilon_{i}=\Delta \epsilon$, which is expressed by $E_{\mathrm{bw}}$ in the figures. The resultant total number of projection orbitals of the monomers is denoted by $N_{\text {proj }}$ throughout the present article.

\section{Initial density matrix: local excitation and electron filling}

The initial density matrices in the GDF representation are prepared so that the diagonal elements in each diagonal block of the corresponding monomer should be occupied up to the number of electrons assigned to this monomer. A simple example can be found in our previous article $\underline{\underline{1}}$ Though an off-diagonal filling responsible for the initial coherence is also possible, for simplicity, we consider only the diagonal part in the preparation of the initial state of the density matrix. This issue will be reported in our future article. In the article, we treat the case of the spin-restricted model within the GDF representation as explained below. This is not rigorously the same as that within the canonical (KS or HF) orbital representation. In a restricted case with the same spatial orbitals for different alpha and beta spins in this model, the occupancy of the GDF local orbitals of each monomer is up to half of the number of electrons assigned to this group from the lowest energy orbital. Therefore, in a strict sense, the initial density matrix mentioned above differs from that of the true ground state of the whole 
system. In fact, this does not cause any problem for examining the migration dynamics of charge and electronic excitations over the constituent monomers in an assembly $\underline{\underline{1}}$

Generally, we can make any type of excitation configuration starting from the reference occupations of the GD orbitals. If we want an initial density associated with an excess or a deficiency of electrons in each monomer for treating the case accompanied with a charge moiety, we merely need to set the occupations to the corresponding number of electrons in each monomer $\underline{\underline{1}}$ Throughout this article, we treat cases with an overall singlet spin state in a spin-restricted manner.

Appendix Aprovides a practical example for preparing an initial density matrix within GD representation.

\section{E. Time propagation of the density matrix in a GD representation}

In this study, the calculation of the time propagation of an electronic state is performed in terms of the Liouvillevon Neumann equation associated with one particle density matrix as follows:

$$
\frac{\partial}{\partial t} \underline{\underline{\rho}}^{\mathrm{GD}}=-\frac{i}{\hbar}\left[\underline{\underline{\mathcal{F}}}^{\mathrm{GD}}\left\{\underline{\underline{\rho}}^{\mathrm{GD}}(t), t\right\}, \underline{\underline{\rho}}^{\mathrm{GD}}\right]
$$

where

$$
\underline{\underline{\mathcal{F}}}^{\mathrm{GD}} \equiv \underline{\underline{F}}^{\mathrm{GD}}+\underline{\underline{L}}^{\mathrm{GD}}
$$

$\underline{\underline{L}}^{\mathrm{GD}}$ is a light-electron coupling matrix. For the length gauge, their corresponding matrix elements have forms of $\underline{\underline{L}}^{\mathrm{GD}}=+e \underline{\underline{\mathbf{r}}}^{\mathrm{GD}} \mathbf{E} \stackrel{25,26}{2}$ where $\mathbf{E}$ is the three-dimensional electric fiel $\overline{\mathrm{d}}$ vector, which generally depend on a point in a three-dimensional space. We used the dipole approximation, namely, long wavelength approximation, $\stackrel{25.26}{=}$ since the wavelength of light treated here is sufficiently large compared to the size of the molecular system treated.

In the RT-TDDFT, the Fock matrix depends on the time-dependent density matrix. For convenience of a discussion on the numerical demonstration to be presented in the later section, we label the dynamics described by Eq. (8) including this dependency as 'RT' meaning 'real time Fock matrix' while the term 'FF' denoting 'frozen Fock matrix' is used to refer to the approximated dynamics with the replacement of the time dependent Fock by that at the initial simulation time. For a technical simplicity and focusing on the projection scheme of electron dynamics, we employ the pure density functional for including an electronic exchange-correlation throughout this article.

For obtaining the time dependent electron density matrix, we solved the non-linear Liouville-von Neumann equation associated with the RT-TDDFT and introduced LP-GDF matrix by using the following two types of numerically stable time integrators, namely (1) the predictor-corrector second order Magnus scheme with linear Fock extrapolation (PC2M-LF) 27 and (2) exponential propagation with predictor-corrector SCF scheme using final corrector as a resultant density (EPPC1) 28 PC2MLF needs one time of update of Fock matrix for each step while an iteration scheme is applied for EPPC1 until a corrector density is converged. Their details are summarized in Appendix B

We followed a dynamics with time steps of 8 and 20 attosecond using a PC2M-LF and EPPC1 time integrator, respectively. Hereinafter we abbreviate femtosecond to fs and attosecond to as. In many cases with a moderate dynamics of density the use of former scheme is sufficient, while the latter was needed for a stable calculation of absorption spectrum using short laser pulses.

The Fock, electron dipole transition matrices within the $\mathrm{AO}$ representation required for the dynamics calculation were evaluated by using the NTChem 2013 software package $\underline{29}$

We should comment on conservations of properties during dynamics. As a special case, we examined a case starting from a locally excited state without external light field. While within the LP-GD scheme a trace of density matrix is perfectly conserved both for RT-TDDFT and Frozen Fock approximation, an expectation value of Fock operator is conserved only for the latter one. Despite this, in a practical sense, this LPGD RT-TDDFT scheme provides a good result with respect to a convergence to reference data as $\Delta_{\epsilon}$ increases, which will be shown in Sect. III with demonstrations of a userbility of the method through calculations of charge migration dynamics and absorption spectrum.

\section{NUMERICAL DEMONSTRATION}

Here, the size effect of the projected space on the electron dynamics and absorption spectrum are examined by using the LP-GDF electron dynamics method introduced in the previous section. We compare the timedependent behaviors of the Mulliken charge of electron donor molecules for NPTL-TCNE with different initial excitation and continuum light field by varying $E_{\mathrm{bw}}$. The convergence of absorption spectrum for 5UT is also examined in the same manner after showing explicitly the importance of the self-consistency between time-dependent density and the time-dependent Fock matrix during electron dynamics.

With respect to the NPTL-TCNE, readers can find further information in our previous article $\frac{1}{=}$ We validate the efficiency of the present projection method by showing a convergence of result with respect to a reduced size of local orbital space. The schematics of the molecular configurations used in this article as shown in Fig. 1.

We also provide information related to the dependence of the computational cost in the electron dynamics calculations with and without Fock build on the size of the projection space, which are detailed in Appendix $\mathrm{F}$. 
(a)

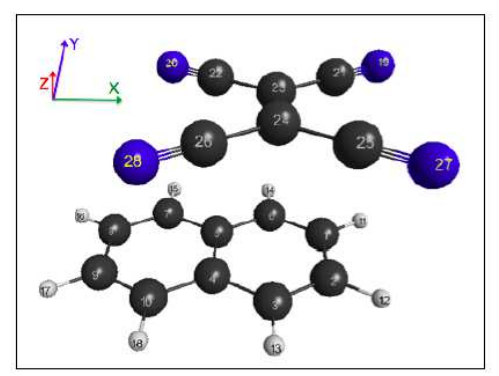

(b)

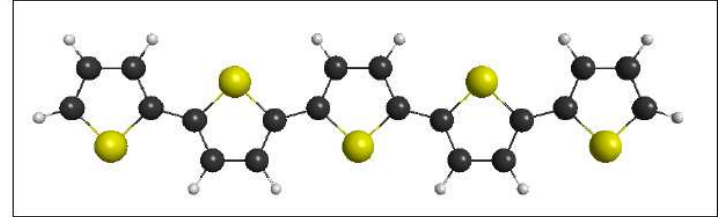

FIG. 1. Schematics of the geometries of the molecular aggregates treated in this article: (a) NPTL-TCNE dimer (b) $5 \mathrm{UT}$ The centroids of aggregates were set to origin for all the systems. See the supplementary material for detailed information of their Cartesian coordinates.

\section{A. NPTL-TCNE dimer}

As a first test system, we treat a dimer system consisting of NPTL and TCNE. In this combination of monomers, NPTL serves as an electron donor molecule, while TCNE plays role as an electron acceptor. The molecular geometries and relative orientations of the monomers used here are the same as those in Panel (1) in Fig. 3 in our previous article $\frac{1}{\underline{*}}$ on the original GDF electron dynamics scheme where readers can find further information including the literature of experimental data.

The geometry of each monomer was optimized at the DFT/6-31G(d) level with the use of the PBE exchange correlation functional 30 The Fock matrix associated with initial optimized KS orbitals and its following time-dependent density matrix for the construction of the GDF matrix was also calculated at the same ab initio level. Both molecules have planar geometries in the optimized geometry in their ground electronic states. Here, as shown in Fig. 11 the principal axis of NPTL was set to be parallel to the $\mathrm{X}$ axis, while we set TCNE to be parallel to the $\mathrm{Y}$ axis. The molecular planes of these flat molecules are parallel to the $\mathrm{X}-\mathrm{Y}$ plane. They were placed in a parallel orientation with a slide of $1.24 \AA$ along the $\mathrm{Y}$ axis. The distance between molecular planes was fixed at $4 \AA$. Although a dimer is treated here, we considered the crystal data 31 reported in the literature with respect to the relative orientation. This selection of molecular configuration yields a non-vanishing overlap between the frontier orbitals, i.e., the HOMO of NPTL and the LUMO of TCNE $\underline{1}$ See the supplementary material for a further information on the geometrical coordinate of this system as well as GD and canonical orbital energies.
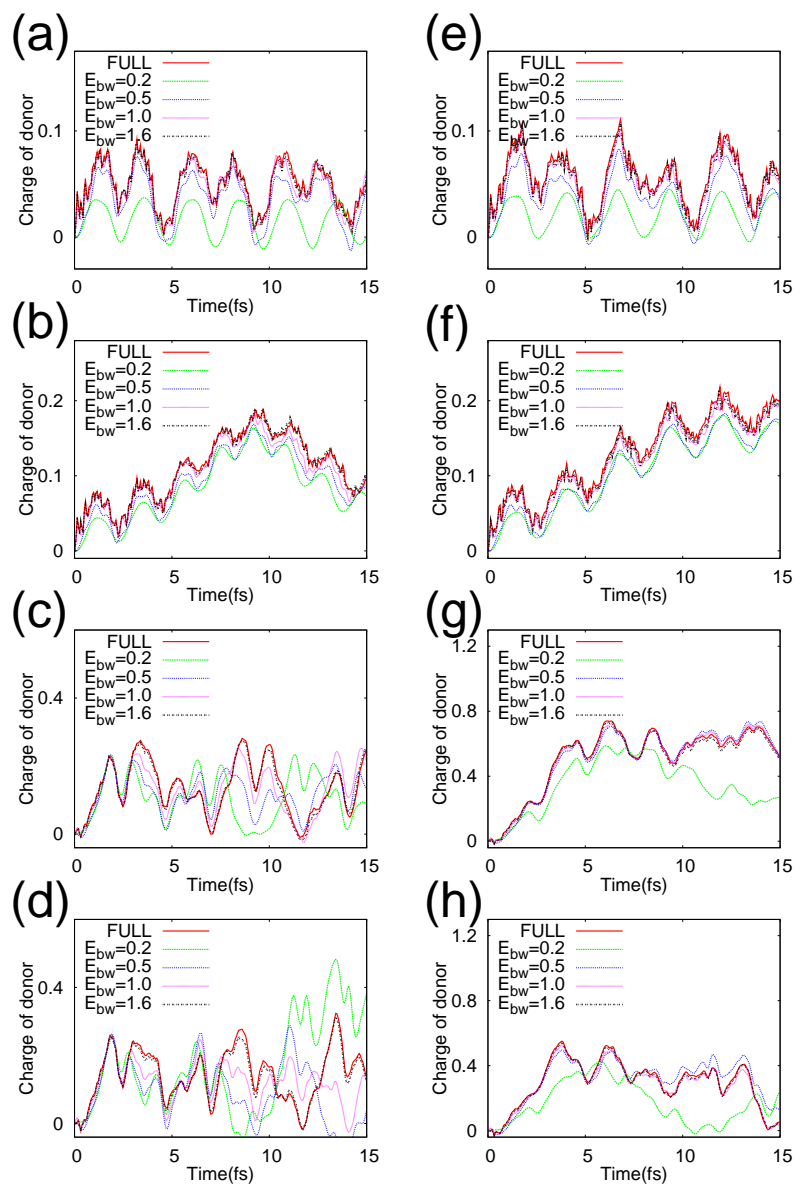

FIG. 2. Convergence of the results for the charge separation dynamics in an NPTL-TCNE dimer with an increase in the energy range determining the projected local orbital space. The energy width used for orbital projection is expressed by $E_{\mathrm{bw}}=\Delta \epsilon$ in Hartree. The numbers of projected orbitals, $N_{\text {proj }}$, are $13,46,74$ and 120 respectively for $\mathrm{E}_{b w}=0.2,0.5$, 1.0 and 1.6. The exact results obtained by using the full 296 orbitals are indicated by solid red line. See the main text for the details of situations with respect to the treatment of Fock matrix, initial local excitation and light field employed in the panels.

Fig. 2 provides the results of the excited electron dynamics involving initial local excitations and external light fields. The time dependent behaviors of the Mulliken charge of the donor molecule, NPTL, are displayed with the variation of the energy range covering the projection orbital space $\Delta \epsilon$. In the panels of the figure, $\Delta \epsilon$ is expressed as $E_{\mathrm{bw}}$. In panels, we plotted the Mulliken charge of NPTL as an electron donor system for each case corresponding to the vertical axis. The horizontal axis denotes the time in femtoseconds.

The results of RT-TDDFT LP-GDF scheme are displayed in panels of $(\mathrm{a}-\mathrm{d})$ while corresponding results of frozen Fock approximation are presented in $(\mathrm{e}-\mathrm{h}) .(\mathrm{a} / \mathrm{e})$ and $(b / f)$ show the results starting from the initial local excitation respectively in the donor NPTL and accep- 
tor TCNE, while the other moieties are initially in the ground states within the GDF representation. In these four panels, no light field is irradiated to the system. In panels $(\mathrm{c} / \mathrm{g})$ and $(\mathrm{d} / \mathrm{h})$, a continuum light field is applied, and its field parameters as a wave length and unit vector of polarization are $700 \mathrm{~nm}$ corresponding to $\omega=0.065$ au and $\left(\frac{1}{\sqrt{3}}, \frac{1}{\sqrt{3}}, \frac{1}{\sqrt{3}}\right)$ in the XYZ Cartesian coordinate, respectively. The field strength $E s$ used in the cases of panels (c/g) is 0.015 a.u., while a larger strength of 0.02 is applied in $(\mathrm{d} / \mathrm{h})$. The function form of light field is given in Appendix E The continuum light field was replaced by a pulse with a sufficiently large time width $t_{w}=2.4 \times 10^{4}$ fs and peak time $t_{c}=0$.

As seen in the RT cases (a) and (b) accompanied with initial local excitations without light fields show the fast convergence with respect to the increase of $E_{\mathrm{bw}}$. In fact, $E_{\mathrm{bw}}=1.0$ associated with 74 projected local orbitals provides a quantitative convergence to the result obtained by the full 296 orbitals. On the contrary, in (c) and (d) from initial local ground states with continuum light field having moderate strength, the quantitative convergence are achieved by $E_{\mathrm{bw}}=1.6$ providing 120 projected orbitals.

As found in the panels $(\mathrm{c} / \mathrm{g})$ and $(\mathrm{d} / \mathrm{h})$, the FF approximation provides faster convergence compared to RT results, corresponding to the cases involved with light fields. On the other hand, the differences of the converged results of FF from those of RT means the importance of a consideration of feedback from time dependent density matrix to the Fock matrix. In (c/d/g/h), we employed the light stronger than that of usual sun light for a severe assessment of the present scheme in cases accompanied with hard excitations. In fact, in this strength, the non-linear effect about the density matrix result in the difference in convergence rates between frozen Fock type and RT-TDDFT calculations with respect to an increase of number of projected orbitals.

To clarify the convergence of time dependent behaviors of charge migration dynamics in Fig. 2 with respect to the number of projected orbitals, we displayed the max deviations of the approximation results measured from the reference data obtained by full orbitals in Fig. 3 as a function of $N_{\text {proj. }}$. As found in the figure, in all cases we can reduce a half the number of the orbitals keeping with the accuracy matched to the result obtained by using full orbitals. The associated CPU times spent for the calculations and the ratios of them to those obtained by the full orbitals are summarized in Appendix $\mathrm{F}$

In the last part of this subsection, as a supplementary information, we provide an evidence of the efficiency of the orbital extraction scheme for the standard RTTDDFT and Frozen Fock approximation by guiding to the corresponding results.

The electron dynamics calculation using the orbital extraction (projection) in standard KS orbital sequence is equivalent to the GDF electron dynamics calculation with $N_{g}=1$, namely no group division. This is because a diagonalization of Löwdin AO representation in case of $N_{g}=1$ gives rise to exactly the same eigen states for (a)

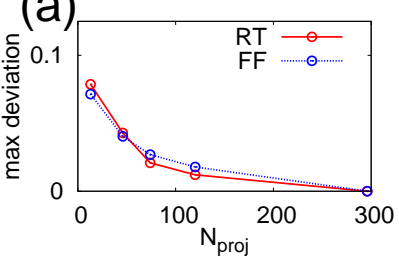

(b)
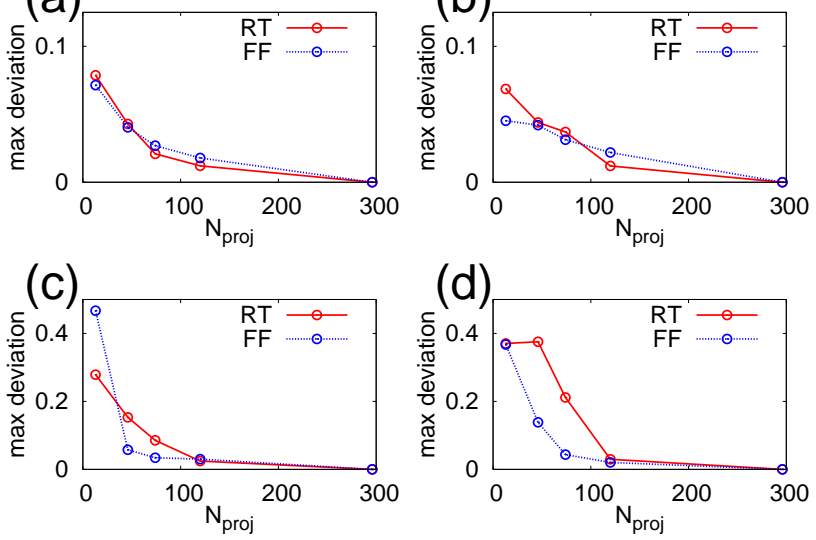

FIG. 3. $\quad N_{\text {proj }}$ dependency of max deviations for donnor molecule during dynamics from the results by full orbital calculation in Fig. 2 Deviation is expressed as an absolute value but not relative one. The panels of (a), (b), (c) and (d) in this figure correspond to those of (a/e), (b/f), (c/g) and (d/h) in Fig. 22 respectively. "RT" and "FF" in panels correspond to the results obtained by electron dynamics calculation using RT-TDDFT scheme considering the self-consistency and frozen Fock approximation.

the original Kohn-Sham equation at an initial simulation time. In the other words, in the case of $N_{g}=1$, total eigen states of Fock operator do not change through a transformation from a representation with original AO to that with Löwdin orthonormalized AOs.

We calculated charge migration of this system by treating the whole system as a monomer, namely, by setting $N_{g}=1$. The energy threshold value for orbital extraction and number of selected orbitals were varied within LP scheme. The data were compared with those obtained by the methods without any orbital projection nor any group division, namely full orbital type of standard RTTDDFT or Frozen Fock approach. Because the setting of $N_{g}=1$ does not allow local excitation of each monomers at initial simulation time in the same sense as $N_{g} \geq 2$, we calculated only the light field cases starting from initial ground state of whole system, namely, no excitation of that. The results and detailed discussion are included in the Supplementary Information.

We should also comment that the Frozen Fock approach without GD representation can provide a better convergence as seen in the comparison between the panel (g) in Fig. 2 and (c) in Fig. S2 with respect to the $E_{\mathrm{bw}}=0.2$ and full orbital cases. In the same sense, RTTDDFT without GD approach can be superior in convergence as found in the comparison between the panel (d) in Fig. 2 and (b) in Fig. S2 for $E_{\mathrm{bw}}=1.0$ and full orbital cases. 


\section{B. 5UT}

In the second demonstration of the present work, we here apply the method to the calculation of absorption spectrum of 5UT. This molecule plays an important role not only as an electron donor but also a hole transfer material in an organic solar cell $\stackrel{2}{2}$ The existence of sulfur atoms in $\pi$ conjugate system gives rise to the stability of molecular structure as well as the increase of charge conduction through the electron overlap contributed by their d-orbitals $\stackrel{33}{ }$ Here we do not discuss a chemical aspect of this system, and just focus on the performance of the method by looking the dependency of results on the size of projected local orbital space.

The molecular geometry of 5UT treated here was optimized in the PBE/6-31G(d) level calculation, which is commonly used in the electron dynamics calculation. The geometry information is summarized in the supplementary materials.

The light pulse was applied and the time-dependent information of induced electronic dipole moments was converted to absorption spectrum, of which details on functional forms are given in Appendix E. We used EPPC1 as the time integrator with the time step of 20as. The total time of simulation carried out was $30 \mathrm{fs}$. The functional form of light field is given in Appendix D The field strength, $E_{s}$, central frequency, $\omega$, pulse peak time, $t_{c}$, pulse width time, $t_{w}$, and angular frequency, $\omega$, of the applied light pulse were 0.001au, $100(=2.42 \mathrm{fs}), 40(=0.97$ fs) and 0.0285 , respectively. The polarization vector of light field was set to be parallel to the vector of $(1,1,1)$ in the Cartesian coordinate. We set the group number $N_{g}=1$. The initial density used in the electron dynamics was set to that of the DFT ground state.

The absorption spectrum evaluated from the formula given in Appendix E numerically depends on the damping parameter, $\gamma$, and the initial cut off time, $T_{\mathrm{CUT}}$. We checked that the simulation time was long enough for the examined energy range from 0 to $4 \mathrm{eV}$.

In order to select a set of reliable parameters for absorption spectrum, we compared the obtained spectrum by varying $\gamma$ and $T_{\mathrm{CUT}}$, which is presented in Fig. 4 including the information of positions of optically-allowed excitations in energy space obtained by LR-TDDFT. The transition properties evaluated by LR-TDDFT including excitation energies, oscillator strength, and dipole moments are summarized in the supplementary material. We also compared the results with and without the feedback of time-dependent density to the Fock matrix in order to show that the instantaneous back-reaction of the density to effective Hamiltonian in the simulation is the key factor for the reproduction of the LR-TDDFT results, which are correspondingly labeled with RT and FF defined in the section of theoretical method, Subsection IIE.

With respect to the parameters, we found the best parameters $\gamma=0.004$ both for $\mathrm{RT}$ and FF cases in the aspects of the balance of proper smoothness and spec- (a) $\gamma=0.006$

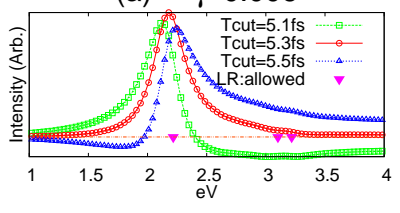

(b) $\gamma=0.004$

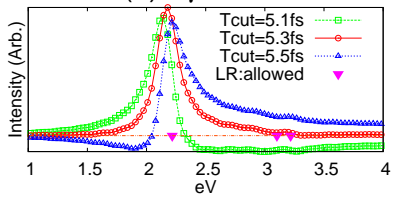

(c) $\gamma=0.002$
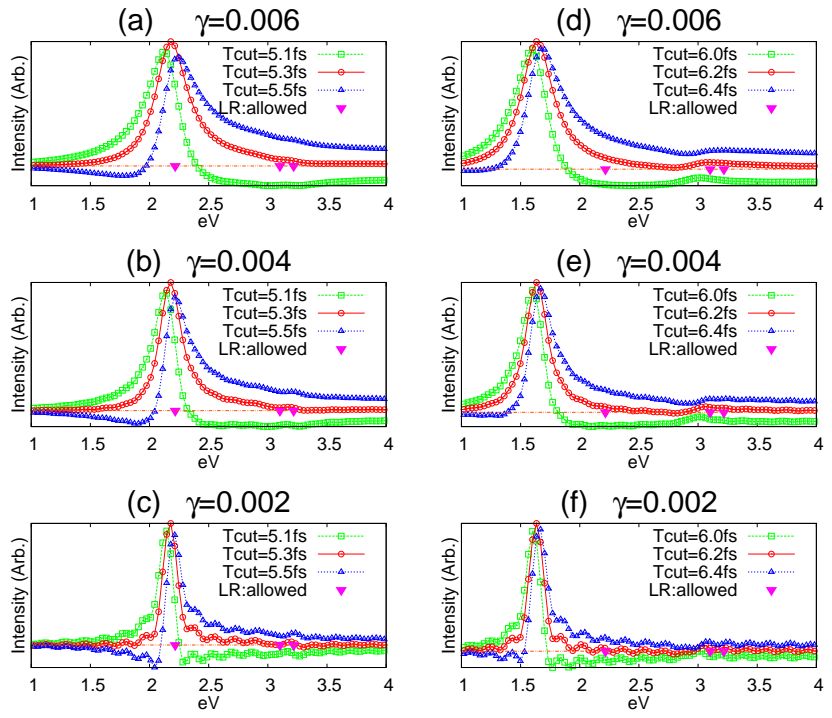

(e) $\gamma=0.004$

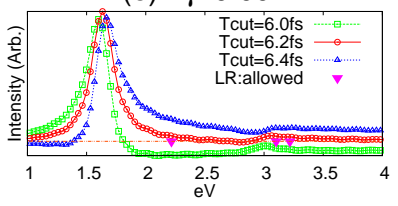

(f) $\gamma=0.002$

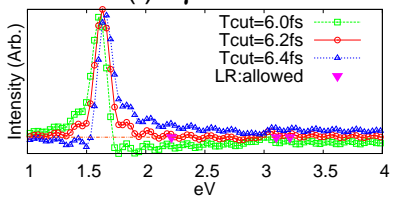

FIG. 4. The importance of feedback of time-dependent information to the Fock matix. We presented absorption spectrums of 5UT with the variation of the damping factor, $\gamma$, and time cut from the initial in the absorption calculation, $T_{\text {CUT }}$. See also the supplementary materials for the mathematical form used for the absorption spectrum. Left panels $(\mathrm{a}-\mathrm{c})$ and right ones $(\mathrm{d}-\mathrm{f})$ correspond to the results of RT and FF, respectively. Note that in all cases here, the full 394 orbitals are used in the dynamics calculations for preparing the light pulse induced electronic dipole moments for the spectrum calculations. Triangles denotes the positions of optically allowed excitations associated with the absorption energy obtained by linear response TDDFT calculations.

trum peak widths. In turn, the best parameters of $T_{\text {CUT }}$ is $5.3 \mathrm{fs}$ and 6.2 for RT and FF based on the observation of the appearance of maximum peaks. Again, we can see that RT calculations presented in the panels ( $\mathrm{a}^{-}$ c) correctly reproduce the first peak position given by LR-TDDFT while FF ones fail. Then, we chose the RT type calculation for spectrum calculation with $\gamma=0.004$ and $T_{\mathrm{CUT}}=5.3$ fs and proceed to check the dependency on the size of projected local orbital space.

Fig. 4 presents the convergence of the absorption spectrum using RT-TDDFT calculation combined with LPGDF scheme with respect to the size of projected local orbital space. We can observe the monotonic convergence of the results by projection scheme to that obtained by the 394 full orbitals. In fact, the spectrum shapes by $E_{\mathrm{bw}}=0.5,0.75$ and 1.5 corresponding to $N_{\text {proj }}=64,89$ and 164 projected orbitals monotonically approaches to that of the full orbital calculation. More than half of the total orbitals were reduced in this spectrum calculation. The information of CPU time is also included in the supplementary material. The success in the reduction of diabatic local orbital space required for the sufficient description suggests the efficiency of the introduced projection method for RT-TDDFT. 


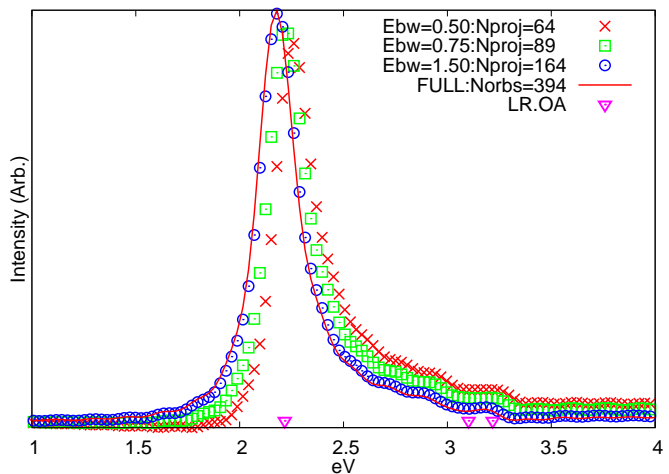

FIG. 5. Projected size dependency and convergence of absorption spectrum. we compared the results by projected local orbitals, $N_{\text {proj }}=64,89$, and 164 correspondingly associated with $E_{\mathrm{bw}}=0.5,0.75$, and 1.5 to that obtained by using $N_{\text {orbs }}=394$ full orbitals for 5 UT molecule. Here we employed the damping factor of $\gamma=0.004$ and $T_{\mathrm{CUT}}=5.3 \mathrm{fs}$ based on the observation in Fig. 4 Triangles denotes the positions of optically allowed excitations associated with the absorption energy obtained by linear response TDDFT calculations, which are labeled with the symbol 'LR.OA'.

\section{CONCLUDING REMARKS}

In this study, we introduced and assessed the electron dynamics method as a combination of the local orbital projection and GDF electron dynamics scheme within a framework of RT-TDDFT. Through the examination of complex charge migration induced by local excitation and light field for the NPTL-TCNE dimer as a test donoracceptor system, we showed that the present method allows us to investigate the size of the Hilbert subspace associated with the excited electron dynamics of molecular aggregates. In the application of the projection method to absorption spectrum, we numerically demonstrated the reproduction of the result with use of the reduced number of orbitals.

We can expect that this method paves a way to a practical investigation of electron dynamics of molecular aggregates with a further combination of the techniques for reducing the cost in Fock build.

We conclude by providing a perspective. The molecular motion, non-adiabatic mixing, and transition between electronic states can affect charge and exciton migration dynamics. There, a nuclear quantum effect may play an important role in electron dynamics in molecular aggregate systems having high density of states. The GD representation scheme can afford a realistic and practical model Hamiltonian for treating these issues. In our future work, we will report on a coarse grained quantum dynamics in molecular aggregates having a nano-size for describing a coupled dynamics of charge and exciton migration associated with their birth, extinction and transport.

\section{SUPPLEMENTARY MATERIAL}

See supplementary material for the issues of (I) validity check of the time interval employed for describing the charge migration dynamics and (II) Efficiency of orbital projection method for standard RT-TDDFT and Frozen Fock method without a group division in charge migration dynamics for NPTL-TCNE system. (III) group diabatic and canonical orbital energies of NPTL-TCNE dimer (IV) optical transition properties of 5UT obtained by LR-TDDFT calculation (V) geometry data of molecular aggregates of a NPTL-TCNE dimer and 5UT.

\section{ACKNOWLEDGMENTS}

This research was supported by MEXT, Japan, "NextGeneration Supercomputer Project" (the K computer project) and "Priority Issue on Post-K Computer" (Development of new fundamental technologies for highefficiency energy creation, conversion/storage and use). Some of the computations in the present study were performed using the Research Center for Computational Science, Okazaki, Japan, and also HOKUSAI system in RIKEN, Wako, Japan.

\section{Appendix A: Initial density matrix within GD representation associated with local excitations}

Essential points in preparing initial density matrix are summarized in a following example. Consider the spinrestricted case with three monomers each of which has 2 electrons and 3 orbitals. Here set $N_{g}=3$. See the main text about the approximate treatment associated with this term of 'spin-restricted'. If we want to prepare the initial condition such that the first and third monomers are initially excited from local HOMO to LUMO, the initial density matrix in GDF scheme is constructed as follows: [1] set reference density matrix, namely GDF ground state, $\underline{\rho}^{\text {GD:ground }}$ and [2] carry out local HOMO LUMO excitation and obtain the aimed GDF density, 
$\underline{\underline{\rho}}^{\mathrm{GD}: 1,3-\mathrm{HL}}$ namely,

$$
\begin{aligned}
& \stackrel{\rho}{\rho}^{\text {GD:ground }} \\
& \Longrightarrow \\
& \underline{\rho}^{\mathrm{GD}: 1,3-\mathrm{HL}} \equiv\left(\begin{array}{ccccccccc}
2 & 0 & 0 & 0 & 0 & 0 & 0 & 0 & 0 \\
0 & 0 & 0 & 0 & 0 & 0 & 0 & 0 & 0 \\
0 & 0 & 0 & 0 & 0 & 0 & 0 & 0 & 0 \\
0 & 0 & 0 & 2 & 0 & 0 & 0 & 0 & 0 \\
0 & 0 & 0 & 0 & 0 & 0 & 0 & 0 & 0 \\
0 & 0 & 0 & 0 & 0 & 0 & 0 & 0 & 0 \\
0 & 0 & 0 & 0 & 0 & 0 & 2 & 0 & 0 \\
0 & 0 & 0 & 0 & 0 & 0 & 0 & 0 & 0 \\
0 & 0 & 0 & 0 & 0 & 0 & 0 & 0 & 0
\end{array}\right) \\
&
\end{aligned}
$$

\section{Appendix B: Time integrator}

We detail the time integrators used for solving nonlinear Liouville von Neumann equation including timedependent Hamiltonian depending on the density matrix employed in this study, namely, (1) the predictorcorrector second order Magnus scheme with linear Fock extrapolation (PC2M-LF) and (2) exponential propagation with predictor-corrector SCF scheme using final corrector as a resultant density (EPPC1). Note again that PC2M-LF needs one time of update of Fock matrix for each step while EPPC1 includes an iteration scheme with respect to a convergence of corrector density. See the main text for the original articles of these schemes.

\section{PC2M-LF}

This scheme consists of the following five processes for each time step,

$$
\begin{aligned}
& \text { [1] } F_{3}:=-\frac{3}{4} F_{1 a}+\frac{7}{4} F_{1 b} \\
& {[2] D_{4}:=e^{-\frac{i}{\hbar} \frac{d t}{2} F_{3}} D_{2} e^{\frac{i}{\hbar} \frac{d t}{2} F_{3}}} \\
& {[3] F_{5}:=F\left[D_{4}\right]} \\
& {[4] D_{6}:=e^{-\frac{i}{\hbar} d t F_{5}} D_{2} e^{\frac{i}{\hbar} d t F_{5}}} \\
& {[5]\left(F_{1 a}, F_{1 b}\right):=\left(F_{1 b}, F_{5}\right) \Rightarrow \text { End of this step }}
\end{aligned}
$$

If we consider a time propagation from $t$ to $t+d t$, $F_{3}$ and $F_{5}$ respectively denote the Fock matrices at $t+$ $\frac{1}{4} d t$ and $t+\frac{1}{2} d t$ while $D_{2}, D_{4}$ and $D_{6}$ correspond to the density matrices at $t, t+\frac{1}{2} d t$ and $t+d t . F_{3}$ in the first step [1] is constructed from the Fock matrices, $F_{1 a}$ and $F_{1 b}$, respectively denote the Fock matrices in the previous two times respectively at $t-\frac{1}{2} d t$ and $t-\frac{3}{2} d t$, by taking a linear extrapolation of them. It is known that owing to the time dividing points set properly this scheme has the same accuracy of second order Magnus expansion. The time propagation matrix having exponential form is treated exactly using a spectrum representation obtained by the diagonalization of Fock matrix, which is also applied in the EPPC1 scheme explained in the next subsection.

\section{EPPC1}

This integrator involved with a micro iteration consists of the following procedures:

$$
\begin{aligned}
& \text { [1] } F_{N}:=F\left[D_{N}\right] \\
& {[2] D_{N+1}^{\text {pred }}:=e^{-\frac{i}{\hbar} d t F_{N}} D_{N} e^{\frac{i}{\hbar} d t F_{N}}} \\
& {[3] F_{N+1}^{\text {pred }}:=F\left[D_{N+1}^{\text {pred }}\right]} \\
& \text { [4] } F^{\text {mid }}:=\frac{1}{2}\left(F_{N}+F_{N+1}^{\text {pred }}\right) \\
& {[5] D_{N+1}^{\text {corr }}:=e^{-\frac{i}{\hbar} d t F^{\text {mid }}} D_{N} e^{\frac{i}{\hbar} d t F^{\text {mid }}}} \\
& {[6]\left\{\begin{array}{c}
\text { If }\left\|D_{N+1}^{\text {corr }}-D_{N+1}^{\text {pred }}\right\|<\epsilon \\
\text { then } D_{N+1}:=D_{N+1}^{\text {corr }} \\
\text { If }\left\|D_{N+1}^{\text {corr }}-D_{N+1}^{\text {pred }}\right\| \geq \epsilon \\
\text { then } D_{N+1}^{\text {pred }}:=D_{N+1}^{\text {corr }}
\end{array} \Rightarrow\right. \text { End of this step }}
\end{aligned}
$$

Here, a time propagation is carried out from $t$ to $t+d t$, which are correspondingly labeled with $N$ and $N+1$. F and $D$ are the Fock and density matrices. 'mid' denotes a mid point of $t$ and $t+d t$, namely, $t+\frac{1}{2} d t$. 'pred' and 'corr' are abbreviations of 'predictor' and 'corrector', respectively. The double 'll' symbol means taking the Frobenius norm of a matrix placed between these two symbols. The threshold is expressed by $\epsilon=n \alpha \xi$ with $n$ being the dimension number of a corresponding matrix. $\alpha$ is set to be a value proportional to the absolute maximum eigen value of the matrix under consideration. Here, we used 1 as $\alpha$ for simplicity. Thus, $\xi$ determines the strictness of the self-consistency between the instantaneous density and the Fock matrix.

\section{Appendix C: Mathematical form of LP-GDF procedure}

Here we provide the formal mathematical expression of LP-GDF scheme. At first, one knows that within the GD representation the identity operator is written as

$$
\hat{1} \simeq \sum_{i=1}^{N_{g}} \sum_{j=1}^{N_{G_{i}}}\left|\phi_{j}^{G_{i}}\right\rangle\left\langle\phi_{j}^{G_{i}}\right|,
$$

where $\left\{\left|\phi_{j}^{G_{i}}\right\rangle \equiv \sum_{k}\left|\widetilde{\chi}_{k, G_{i}}\right\rangle\left[\underline{\underline{D}}_{G_{i}}\right]_{k j}\right\}$ with $\left|\widetilde{\chi}_{k, G_{i}}\right\rangle$ being the k-th Löwdin orthogonalized atomic orbital basis 
function spanned in the $\mathrm{G}_{i}$-th group is the set of GD localized orbitals for the group labeled by $G_{i}$ and $j$ ranges from 1 to $N_{G_{i}}$, which denotes the number of basis functions spanned at the site, $G_{i}$. $i$ ranges from 1 to $N_{g}$, i.e., the number of monomer group sites. We used the symbol for approximation in the equation because of the practical use of a finite basis set during computation. Note that the GDF orbitals created from Löwdin orthonormal basis remain to be orthogonal under unitary transformations even if the transformations are carried out in each group. In turn, with respect to the structure of matrix representation of the Fock operator, only the GDF orbital pairs between different monomers are Fock nonorthogonal while the pairs within the same group are Fock orthogonal .

Next, we introduce projection operators in order to realize steps (i)-(v) in Subsect IC as follows:

$$
\hat{P} \equiv \sum_{i=1}^{N_{g}} \sum_{j \in \Omega_{i}}^{N_{G_{i}}}\left|\phi_{j}^{G_{i}}\right\rangle\left\langle\phi_{j}^{G_{i}}\right|
$$

where

$$
\Omega_{i} \equiv\left\{j ;\left|\epsilon_{j, G_{i}}-\bar{\epsilon}\right| \leq \Delta \epsilon_{i} / 2\right\} \quad \text { and } \quad \bar{\epsilon} \equiv\left(\epsilon_{\mathrm{H}}+\epsilon_{\mathrm{L}}\right) / 2 .
$$

Here, $\epsilon_{j, G_{i}}$ is the local orbital energy associated with the GD orbital $\phi_{j}^{G_{i}}$, while $\epsilon_{\mathrm{H}}$ and $\epsilon_{\mathrm{L}}$ are the HOMO and LUMO energies of the whole system.

By using these mathematical tools within LP-GDF formulation, we approximate a one electron operator $\hat{O}$ as follows:

$$
\hat{O} \approx \hat{O}_{P} \equiv \hat{P} \hat{O} \hat{P}
$$

\section{Appendix D: Function form of Laser pulse field}

The vector potential of light field as a function of time in the long wave length approximation employed takes a form of

$$
\mathbf{A}(t)=\sum_{j}^{N_{\mathrm{p}}} \mathbf{A}_{j} f\left(t ; t_{c_{j}}, t_{w_{j}}\right) \cos \left(\omega_{j}\left(t-t_{c_{j}}\right)+\delta_{j}\right),
$$

where bold font means the three dimensional vector in the Cartesian coordinate space. Here, the envelope function is defined by $f\left(t ; t_{c}, t_{w}\right) \equiv \exp \left(-\left(\frac{t-t_{c}}{t_{w}}\right)^{2}\right)$. The physical meanings of parameters appeared above are as follows; $t$ denotes time, $t_{c}$ is a field peak time, $t_{w}$ stands for a typical gaussian decay time, $\omega$ means a central angular frequency of field, and $\delta$ is a carrier envelope phase. $N_{\text {p }}$ denotes a number of pulses. The electric field vector of external light corresponding to $\mathbf{A}(t)$ is given by $\mathbf{E}(t)=-\frac{1}{c} \frac{\partial \mathbf{A}(t)}{\partial t}$. In the present article, we employed $N_{\mathrm{p}}=1$ and $\delta=0$.

\section{Appendix E: Absorption spectrum}

The absorption spectrum was evaluated using the following function,

$$
S(\omega) \equiv \frac{1}{3} \operatorname{Tr}[\underline{\underline{\sigma}}(\omega)]
$$

where $\underline{\underline{\widetilde{\sigma}}}(\omega) \equiv \frac{4 \pi \omega}{c} \operatorname{Im}[\underline{\underline{\widetilde{\alpha}}}(\omega)]$ is an absorption cross section with $[\underline{\underline{\alpha}}(\omega)]_{j k} \equiv \frac{\widetilde{\mu}_{j}^{\mathrm{ind}}(\omega)}{\widetilde{E}_{k}(\omega)}=\frac{\int_{T_{\mathrm{CUT}}}^{T_{\mathrm{FIN}}} e^{i \omega t} e^{-\gamma t} \mu_{j}(t)}{\int_{T_{\mathrm{CUN}}}^{T_{\mathrm{FIN}}} e^{i \omega t} E_{k}(t)} . \gamma$ is a damping factor. Here, $\mu_{j}^{\text {ind }}(t) \equiv \mu_{j}(t)-\mu_{j}(0)$ and $E_{k}(t)$ are induced dipole moment and external electric field, respectively. The symbol of tilde means taking the Fourier transformation. $T_{\text {FIN }}$ is a final simulation time. $T_{\text {CUT }}$ is the initial time used for a transformation from time dependent induced dipole moment of electrons and external field to absorption spectrum.

\section{Appendix F: Computational time}

Here, we provide information about the computational cost of electron dynamics including analysis of the timedependent properties. Note that the overhead of parallel computation is also included in time. The aim here is to show examples of the performance of the method in cases with use of moderate computer facilities.

The specifications of the computer and compiler utilized in this article are as follows: [For NPTL-TCNE] \{ Intel(R) Xeon(R) Gold 61480 @ $2.40 \mathrm{GHz}$ with a cache size of 27.5MB, The Intel FORTRAN compiler in Version 18.0.2.199 with level three optimization. $\}$ [For 5UT] \{ Intel(R) Xeon(R) Gold 61520 @ 2.10GHz with a cache size of $30.9 \mathrm{MB}$, The Intel FORTRAN compiler in Version 17.0.4.196 with level three optimization. $\}$

In all the calculations $40 \mathrm{cpu}$ cores were used in a parallel manner using openMP for matrix-matrix product and MPI for Fock build if needed.

\section{NPTL-TCNE}

Tab. \and Tab. Пpresent the comparison of the computation times using different number of projected local orbitals in cases of PC2M-LF and EPPC1 methods, respectively. The system treated is the NPTL-TCNE dimer. A ratio to the time spent in cases with full orbitals expresses an acceleration in computation by using projected space. The tables include the comparisons between RT and FF. Note that the time intervals of one time step employed here are different between these two integrators. We checked the reproduction of the same result by using these two integrators, which is presented in the supplementary material.

In Tab. Пfor PC2M-LF, though we can see that a moderate acceleration is attained with a help of projected 


\begin{tabular}{cccc}
\hline$E_{\text {bw }}$ & $N_{\text {proj }}$ & RT & FF \\
\hline 0.2 & 13 & $108[0.14]\{1\}$ & $16[0.03]\{0\}$ \\
0.5 & 46 & $120[0.15]\{1\}$ & $24[0.04]\{0\}$ \\
1.0 & 74 & $141[0.18]\{1\}$ & $40[0.06]\{0\}$ \\
1.6 & 120 & $203[0.26]\{1\}$ & $93[0.14]\{0\}$ \\
FULL & 296 & $801[1.00]\{1\}$ & $679[1.00]\{0\}$ \\
\hline
\end{tabular}

TABLE I. CPU time spent for the dynamics calculations plus the time-dependent analysis for NPTL-TCNE with PC2MLF scheme with 1875 time steps using 8 as time interval. The unit of time is second. Numeric value in [] denotes the ratio to the cost in case with use of full orbitals while that in \{\} mean the number of Fock build per one time step. Note the single Fock build per one time step in the PC2M-LF scheme. The spent time needed in the cases of Fig. 2 are the same in each group of the panels (a-d) for RT and (e-h) for FF, namely, in the above RT and FF columns correspond to the group of (a-d) and (e-h) in Fig. 2. See the text for the meanings of $E_{\mathrm{bw}}=\Delta \epsilon, N_{\text {proj }}, \mathrm{RT}$ and FF. The wall time means the wall clock time associated with openMP for dynamics and MPI for Fock build. space and acceleration in FF is superior to RT one including Fock build, the use of RT recommended for the reliable calculation and provides still moderate acceleration by projections of local orbital space. In fact, the computation time in RT with $E_{\mathrm{bw}}=1.6$ corresponding to $N_{\text {proj }}=120$ which provide converged results is reduced to one fourth of that spent in the cases using the full orbitals.

As seen in Tab. II the acceleration in the calculation by projection in case of EPPC1 including micro iterations accompanied with Fock build for a convergence of corrector density is less than those of PC2M-LF in Tab. [1] Despite of this, the case of $E_{\mathrm{bw}}=1.6$ with $N_{\text {proj }}=120$ need approximately the half of the computational time in the full orbital case.

\begin{tabular}{cccccc}
\hline$E_{\text {bw }}$ & $N_{\text {proj }}$ & $(\mathrm{a})$ & $(\mathrm{b})$ & $(\mathrm{c})$ & $(\mathrm{d})$ \\
\hline 0.2 & 13 & $185[0.17]\{2.6\}$ & $155[0.14]\{2.0\}$ & $229[0.20]\{3.1\}$ & $256[0.21]\{3.4\}$ \\
0.5 & 46 & $239[0.21]\{3.5\}$ & $267[0.24]\{4.0\}$ & $310[0.26]\{4.0\}$ & $332[0.27]\{4.0\}$ \\
1.0 & 74 & $302[0.27]\{4.0\}$ & $312[0.28]\{4.2\}$ & $350[0.29]\{4.1\}$ & $360[0.29]\{4.1\}$ \\
1.6 & 120 & $621[0.56]\{5.0\}$ & $457[0.41]\{5.0\}$ & $534[0.45]\{5.0\}$ & $545[0.44]\{5.0\}$ \\
FULL & 296 & $1121[1.00]\{6.1\}$ & $1132[1.00]\{6.2\}$ & $1205[1.00]\{6.2\}$ & $1256[1.00]\{6.7\}$ \\
\hline \hline$E_{\text {bw }}$ & $N_{\text {proj }}$ & $(\mathrm{e})$ & $(\mathrm{f})$ & $(\mathrm{g})$ & $(\mathrm{h})$ \\
\hline 0.2 & 13 & $8[0.04]\{1\}$ & $8[0.04]\{1\}$ & $8[0.03]\{1\}$ & $8[0.03]\{1\}$ \\
0.5 & 46 & $11[0.05]\{1\}$ & $11[0.05]\{1\}$ & $11[0.04]\{1\}$ & $11[0.04]\{1\}$ \\
1.0 & 74 & $17[0.07]\{1\}$ & $17[0.07]\{1\}$ & $17[0.07]\{1\}$ & $17[0.07]\{1\}$ \\
1.6 & 120 & $38[0.14]\{1\}$ & $39[0.15]\{1\}$ & $39[0.14]\{1\}$ & $38[0.14]\{1\}$ \\
FULL & 296 & $274[1.00]\{1\}$ & $271[1.00]\{1\}$ & $283[1.00]\{1\}$ & $283[1.00]\{1\}$ \\
\hline
\end{tabular}

TABLE II. CPU time spent for the dynamics calculations plus the time-dependent analysis for NPTL-TCNE with EPPC1 scheme with 750 time steps using 20 as time interval. The unit of time is second. Numeric value in [] denotes the ratio to the cost in case with use of full orbitals while that presented in \{\} correspond to the average number of cycles in micro-iterations needed for the density convergence with $\xi=10^{-8}$. See the text for the meanings of $E_{\mathrm{bw}}=\Delta \epsilon, N_{\text {proj }}, \mathrm{RT}$ and FF. (a-d) and (e-h) correspond to RT and FF, respectively, and all the symbols are the same as Fig. 2 in the main text. The definition of the wall time is the same as that in Tab. I]

\section{2. $5 \mathrm{UT}$}

Tab. III presents the comparison of the computation times using different number of projected local orbitals in cases of EPPC1 methods for 5UT system. The way of presentation is the same as the table for NPTL-TCNE. Here also we show the comparisons between RT and FF. As seen in Tab. III the case of $E_{\mathrm{bw}}=1.5$ with $N_{\text {proj }}=$ 164 associated with the converged spectrum presented in Fig. 5 in the main text needs merely less than half of the computational time in the case with the full 394 orbitals. 


\begin{tabular}{ccc}
\hline$E_{\mathrm{bw}}$ & $N_{\text {proj }}$ & CPUtimes \\
\hline 0.5 & 64 & $51949[0.43]\{2.8\}$ \\
0.75 & 89 & $53447[0.44]\{2.9\}$ \\
1.5 & 164 & $61075[0.50]\{2.9\}$ \\
FULL & 394 & $123033[1.00]\{3.8\}$ \\
\hline
\end{tabular}

TABLE III. CPU time spent for the dynamics calculations plus the time-dependent analysis for 5UT with EPPC1 scheme with 1500 time steps using 20as time steps. The unit of time is second. Numeric value in [] denotes the ratio to the cost in case with use of full orbitals while that presented in \{\} correspond to the average number of cycles in micro-iterations needed for the density convergence with $\xi=10^{-8}$. See the text for the meanings of $E_{\mathrm{bw}}=\Delta \epsilon$ and $N_{\text {proj. }}$. The wall time means the wall clock time, and the values in parentheses denote the CPU efficiency in percent associated openMP for dynamics and MPI for Fock build. 
* takehiro.yonehara@riken.jp

$\dagger$ nakajima@riken.jp

1 T. Yonehara and T. Nakajima, J. Chem. Phys. 147 (2017) 074110 .

${ }^{2}$ I. Kondov, Martin Cizek, C.Benesch, H. Wang, and M. Thoss, J. Phys. Chem. C 111 (2007) 11970.

3 T. Pacher, L. S. Cederbaum, and H. Köppel, J. Chem. Phys. 89 (1988) 7367.

4 C. Benesch, M. Cizek, M. Thoss, and W. Domcke, Chem. Phys. Lett. 430 (2006) 355.

${ }^{5}$ V. Mujica, M. Kemp, and M. A. Ratner, J. Chem. Phys. 101 (1994) 6849.

6 Time-Dependent Density Functional Theory (Lecture Notes in Physics) M. A.L. Marques, C. A. Ullrich, F. Nogueira, A. Rubio, K. Burke, E. K. U. Gross ( Springer, 2006 ).

7 Fundamentals of Time-Dependent Density Functional Theory M. A. Marques, N. T. Maitra, F. M. Nogueira, E. K. U. Gross, and A. Rubio, ( Springer, 2012 ).

8 Time-Dependent Density-Functional Theory: Concepts and Applications C. A. Ullrich, ( Oxford Univ Press, 2012 ).

${ }^{9}$ Real-time time-dependent electronic structure theory J. J. Goings, P. J. Lestrange, X. Li, Wiley Interdisciplinary Reviews : Computational Molecular Science Volume 8 Issue 1 ( Springer, 2012 ).

10 J. Li, I. Kondov, H. Wang, and M. Thoss, J. Phys. Chem. C 114 (2010) 18481.

11 Y. Xie, J. Zheng, and Z. Lan, J. Chem. Phys. 142 (2015) 084706.

12 A. Cembran, L. Song, Y. Mo, and J. Gao, J. Chem. Theory Comput. 5 (2009) 2702.

13 B. Shi, F. Gao, and W. Liang, Chem. Phys. 394 (2012) 56.

14 A. F. Morrison, Z.-Q. You, and J. M. Herbert, J. Comp. Theoret. Chem. 10 (2014) 5366.

${ }^{15}$ K. J. Fujimoto, J. Chem. Phys. 137 (2012) 034101.

16 K. J. Fujimoto and W. Yang, J. Chem. Phys. 129 (2008) 054102 .

17 H. Oberhofer, K. Reuter, and J. Blumberger, Chem. Rev. 117 (2017) 10319.

18 A. V. Akimov, and O. V. Prezhdo J. Am. Chem. Soc. 136 (2014) 1599

19 L. Wang, O. V. Prezhdo, and D. Beljonne Phys. Chem. Chem. Phys. 17 (2015) 12395.

20 J. Spencer, F. Gajdos, and J. Blumberger J. Chem. Phys. 145 (2016) 064102.

21 A. Carof, S. Giannini, and J. Blumberger J. Chem. Phys. 147 (2017) 214113.

22 A. Heck, J. J. Kranz, and M. Elstner J. Chem. Theory Comput. 12 (2016) 3087.

23 Z. Futera and J. Blumberger J. Phys. Chem. C 121 (2017) 19677.

${ }^{24}$ A. Szabo and N. S. Ostlund, Modern Quantum Chemistry: Introduction to Advanced Electronic Structure Theory (Dover, New York, 1996).

25 F. H. M. Faisal, Phys. Rev. A 75 (2007) 063412.

${ }^{26}$ K. Takatsuka and T. Yonehara, Adv. Chem. Phys. 144 (2010) 93

27 C.-L. Cheng, J. S. Evans, and T. V. Voorhis, Phys. Rev. B 74 (2006) 155112.

${ }^{28}$ Y. Zhu and J. M. Herbert, J. Chem. Phys. 148 (2018) 044117.
29 T. Nakajima, M. Katouda, M. Kamiya, and Y. Nakatsuka, Int. J. Quantum Chem. 115 (2015) 349.

30 A. Zupan, K. Burke, M. Ernzerhof, and J. P. Perdew, J. Chem. Phys. 106 (1997) 10184; J. P. Perdew, M. Ernzerhof, A. Zupan, and K. Burke, J. Chem. Phys. 108 (1998) 1522.

31 R. M. Williams and S. C. Wallwork, Acta. Cryst. 22 (1967) 899.

32 T. P. Kaloni, P. K. Giesbrecht, G. Schreckenbach, and M. S. Freund, Chem. Mater. 29 (2017) 10248.

${ }^{33}$ T.-C. Chung, J. H. Kaufman, A. J. Heeger, and F. Wudl Phys. Rev. B 30 (1984) 702. 
Supplementary material for "Electron dynamics method using a locally projected group diabatic Fock matrix for molecule and aggregate"

I: Convergence of charge migration dynamics with respect to the time increment using different integrator for NPTL-TCNE system.

Fig. S1 shows the convergence of charge migration dynamics for NPTL-TCNE system with respect to time increments. The panels correspond to those in Fig. 2 in the main article. For a stringent check, we used different integrators, namely, PC2M-LF and EPPC1, with the time increments of 8 and 20 as in each step, respectively. The perfect agreement with each other means the convergence of the result.
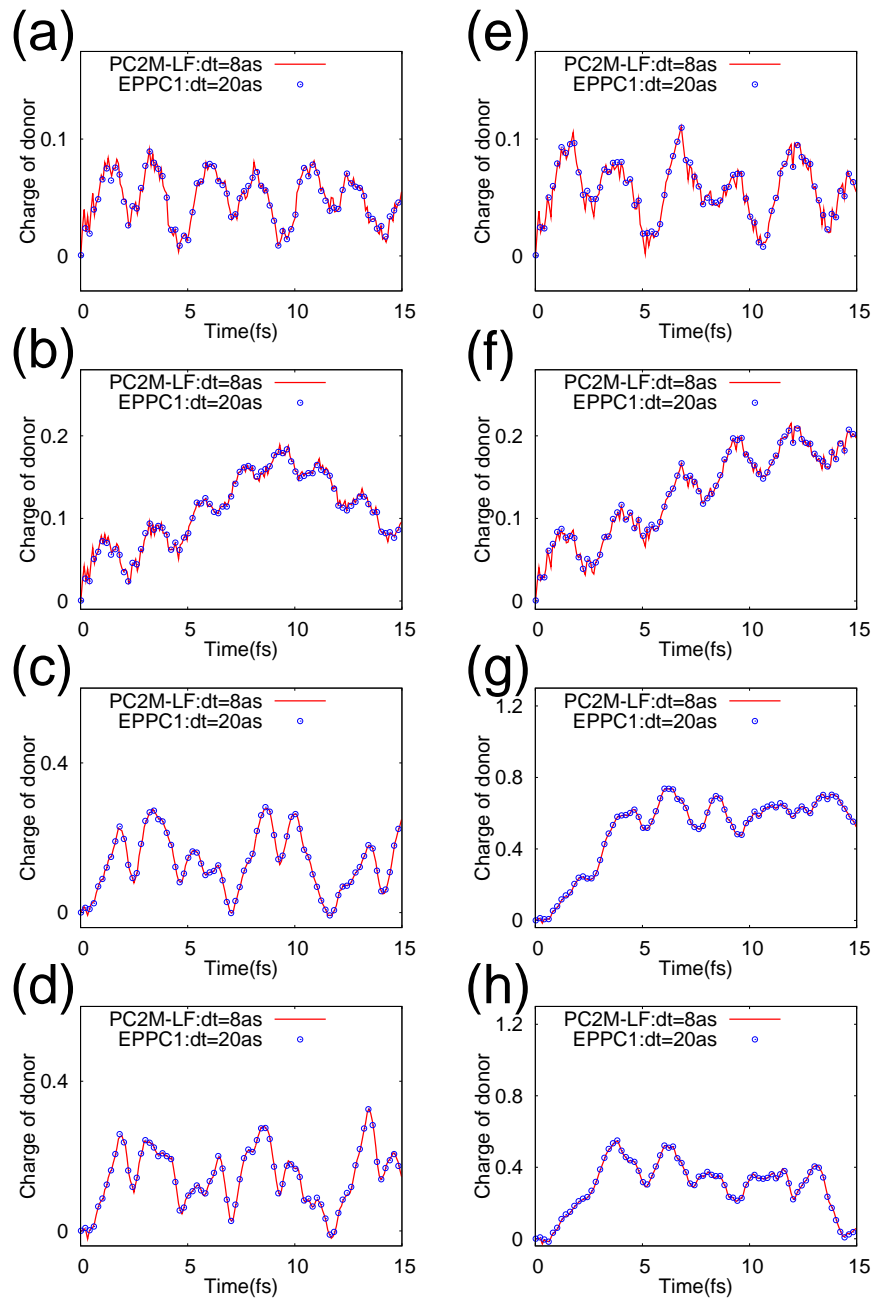

FIG.S 1. Check of the convergence of charge migration dynamics with respect to the time increment using different integrator for NPTL-TCNE system. See the main text in this supplementary material about the panels. 


\section{II: Efficiency of orbital projection method for standard RT-TDDFT and Frozen Fock method without a group division in charge migration dynamics for NPTL-TCNE system.}

We demonstrate an efficiency of the present local projection (LP) scheme for a standard RT-TDDFT. Note that the standard RT-TDDFT is equivalent to the case with $N_{g}=1$ and a ground state of whole system as an initial state within LP-GDF electron dynamics scheme.

This is also verified for the calculation of absorption spectrum for $5 \mathrm{UT}$ with $N_{g}=1$, which is presented in Fig. 5 in the main text. Here, for charge migration dynamics in NPTL-TCNE system, we show the efficiency of LP scheme for the standard RT-TDDFT without any group division in the Fock matrix. We also show the results for the Frozen Fock approach.

In this context, panels $(\mathrm{a} / \mathrm{b})$ and $(\mathrm{c} / \mathrm{d})$ in Fig. $\mathrm{S} 2$ with $N_{g}=1$ in LP-GDF electron dynamics correspond to (c/d) and $(\mathrm{g} / \mathrm{h})$ of Fig. 2 in the main text with $N_{g}=2$, respectively. The same properties are plotted. Time integrator employed here is EPPC1. Strictly speaking, the initial electronic condition is different for both schemes since the former standard case starts from the initial density matrix made from the ground state of whole system while the initial density matrix was prepared by using the combination of local ground states of monomers in the latter LP-GDF case. Despite this, the time dependent behavior is similar for both cases, which inversely supports validity of group division in Fig. 2.

Fig. 93 is the maximum error analysis for the LP results compared from the full orbital calculations. Panels (A) and (B) correspond to the cases of (a/b) and (c/d) in Fig. S2 The plots of the data were carried out in the same way as Fig. 3 in the main text.

These results indicate that the LP scheme can safely reduce the computational cost with respect to orbital numbers required for the description also for charge migration dynamics under a light field with using standard RT-TDDFT equivalent to LP-GDF-electron dynamics scheme with $N_{g}=1$.
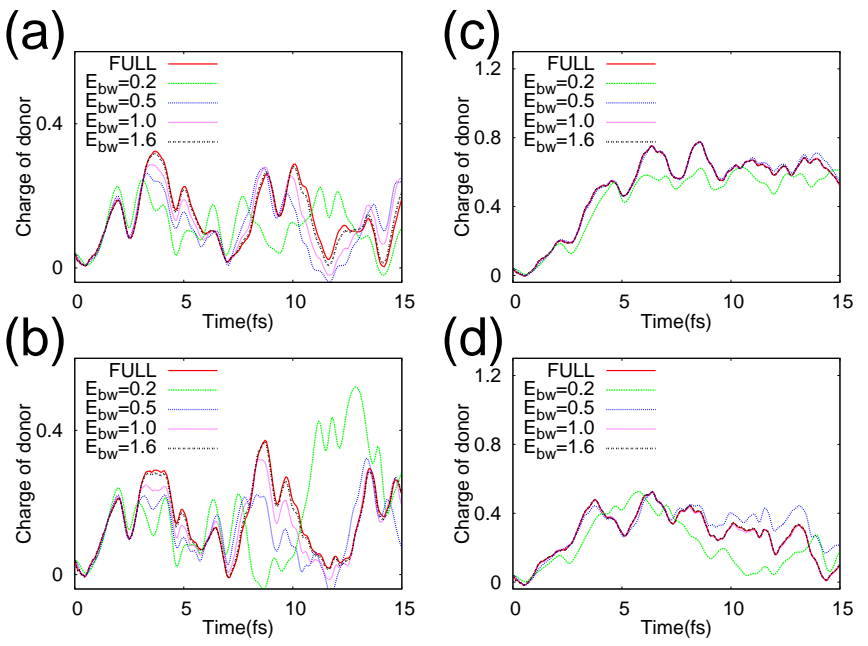

FIG.S 2. Light induced charge migration dynamics with $N_{g}=1$ for NPTL-TCNE. Panels (a), (b), (c) and (d) in this figure respectively correspond to (c), (d), (g) and (h) in Fig. 2 in the main text.
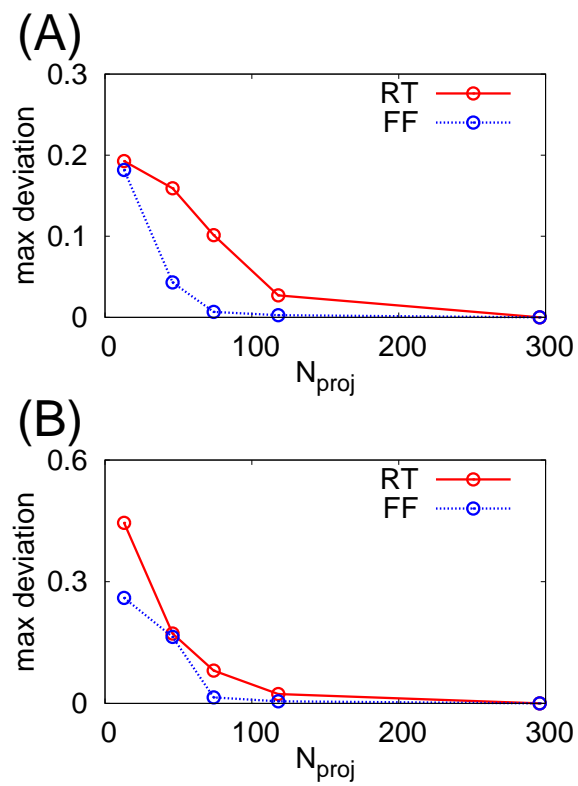

FIG.S 3. $\quad N_{\text {proj }}$ dependency of max deviations for donor molecule during dynamics from the results by full orbital calculation in Fig. \$2 RT and FF in panel (A) were evaluated from the results in (a) and (c) of Fig. $\mathrm{S} 2$ while RT and FF in panel (B) were evaluated from the results in (b) and (d). 


\section{III: Group diabatic and canonical orbital energies of NPTL-TCNE dimer}

Tab. [1)summarizes the energy differences of LUMO+1, LUMO and HOMO-1 measured from HOMO, which are presented for group diabatic and canonical representations associated with monomers and dimer system, respectively.

\section{IV: Excitation energies, transition dipoles and oscillator strength of 5UT}

Tab.SII summarizes the information on lower excitations of $5 \mathrm{UT}$ at the geometry given in the later section in this supplementary material. We showed the excitation energies, transition dipole moment vectors, and oscillator strength.

Here, $(i \rightarrow j), \Delta E_{i j} \equiv\left|E_{i}-E_{j}\right|, \vec{L}_{i j}$ and $f_{i j} \equiv$ $\frac{2}{3}\left(\Delta E_{i j}\right)\left|\vec{L}_{i j}\right|^{2}$ denote a state pairs of transition, energy difference of them, transition dipole moment vector for them, and its corresponding oscillator strength, respectively. $E_{i}$ means $i$-th adiabatic state with 0 being ground electronic state. The geometry of this molecular system is given later in this supplementary material. According to magnitudes of oscillator strength, we considered that $0 \rightarrow 1,0 \rightarrow 3$ and $0 \rightarrow 5$ are optically allowed transitions, and used as reference excitation energies in the presentation of Fig. 4 and 5 in the main text.

Though it is not the aim to reproduce the experimental data in the present article since the main focus is the projection scheme in electron dynamics calculation, we provide information near to the situation treated here

\begin{tabular}{|l|c|c|}
\hline $\mathrm{GD}$ & $(\mathrm{D}) \mathrm{NPTL}$ & $(\mathrm{A}) \mathrm{TCNE}$ \\
\hline$\Delta \epsilon_{\mathrm{L}+1, \mathrm{H}}^{\mathrm{G}}$ & +0.1554 & +0.1925 \\
$\Delta \epsilon_{\mathrm{L}, \mathrm{H}}^{\mathrm{G}}$ & +0.1244 & +0.0939 \\
$\Delta \epsilon_{\mathrm{H}, 1-\mathrm{H}}^{\mathrm{G}}$ & -0.0312 & -0.0182 \\
\hline \multicolumn{1}{|c|}{$\mathrm{CA}$} & NPTL-TCNE \\
\hline$\Delta \epsilon_{\mathrm{L}+1, \mathrm{H}}^{\mathrm{W}}$ & +0.1247 \\
$\Delta \epsilon_{\mathrm{L}, \mathrm{H}}^{\mathrm{W}}$ & +0.0586 \\
$\Delta \epsilon_{\mathrm{H}-1, \mathrm{H}}^{\mathrm{W}}$ & -0.0303 \\
\hline
\end{tabular}

TABLE.S I. (Upper table) Energy differences of group diabatized HOMO-1, LUMO, and LUMO+1 measured from group diabatized HOMO of the electron donor NPTL and acceptor TCNE moieties in a molecular complex, which are labeled with the symbols of $\Delta \epsilon_{\mathrm{H}-1, \mathrm{H}}^{\mathrm{G}}, \Delta \epsilon_{\mathrm{L}, \mathrm{H}}^{\mathrm{G}}$, and $\Delta \epsilon_{\mathrm{L}+1, \mathrm{H}}^{\mathrm{G}}$, respectively. Note that the monomer interactions are included in the evaluations of the local orbital energies of monomers. (Lower table) Energy differences of canonical HOMO-1, LUMO and LUMO+1 measured from HOMO for the whole NPTL-TCNE dimer system, which are denoted by the symbols of $\Delta \epsilon_{\mathrm{H}-1, \mathrm{H}}^{\mathrm{W}}$, $\Delta \epsilon_{\mathrm{L}, \mathrm{H}}^{\mathrm{W}}$, and $\Delta \epsilon_{\mathrm{L}+1, \mathrm{H}}^{\mathrm{W}}$, respectively. In both tables, the unit is atomic unit. GD and CA mean 'group diabatic' and 'canonical', respectively. with respect to the target system. The experimental data of the position of the first peak in absorption spectrum of optical electronic transition for this 5UT system, however, in the $\mathrm{CHCl}_{3}$ solvent but not in a gas phase, is reported in the former works, $\frac{1}{=}$ and its value is $2.98 \mathrm{eV}$. Though the absorption spectrum for electronic transition in a molecular system can be changed by an existence of a polar solvent associated with a complex modification of electric transition and structure relaxation, this is not significant in this system.

On the other hand, as seen in Fig. 4 of the main article as the comparison part of RT and FF results, the value given by RT-TDDFT, $2.2 \mathrm{eV}$, being consistent with LRTDDFT result here within the present limited ab initio level is close to the experimental value mentioned above, compared to the peak value, $1.6 \mathrm{eV}$, given by $\mathrm{FF}$ approximation as found in Fig. 4. This observation also supports the basic assertion of the importance of the self-consistent treatment for the Fock matrix and time-dependent density matrix in a calculation of absorption spectrum.

The remaining discrepancy in the LR-TDDFT result here is remedied by using more appropriate higher level basis set $\underline{\underline{1}}$ and exchange-correlation functionals ${ }^{2}$.

In fact, at the same structure, the use of the representative hybrid functionals with a long-range correction, namely, CAM-B3LYP 3 and $\mathrm{LC}^{-\mathrm{BOP}^{4}}{ }^{4}$, provide the optically allowed first electronic transition energies in gas phase, $2.80 \mathrm{eV}$ and 2.99 , respectively, while their corresponding values for the polarizable continuum model $(\mathrm{PCM})^{\frac{5}{5}}$ with $\mathrm{CHCl}_{3}$ solvent at the same structure are 2.68 and 2.88. In the calculations using the PCM combined with LR-TDDFT here, we used the ab initio program package for electronic structure calculation, GAMESS.$\underline{6}$

\begin{tabular}{|c|c|r|r|r|c|}
\hline$(i \rightarrow j)$ & $\Delta E_{i j}(\mathrm{eV})$ & \multicolumn{3}{|c|}{$\vec{L}_{i j}(\mathrm{au})$} & $f_{i j}(\mathrm{au})$ \\
\hline \hline $0 \rightarrow 1$ & 2.22 & +5.11 & +0.00 & +0.00 & 1.42 \\
$0 \rightarrow 2$ & 2.37 & +0.00 & -0.01 & +0.00 & 0.00 \\
$0 \rightarrow 3$ & 3.10 & -1.16 & +0.00 & +0.00 & 0.10 \\
$0 \rightarrow 4$ & 3.18 & +0.00 & -0.13 & +0.00 & 0.00 \\
$0 \rightarrow 5$ & 3.22 & +1.64 & -0.00 & +0.00 & 0.21 \\
$0 \rightarrow 6$ & 3.57 & +0.00 & -0.06 & +0.00 & 0.00 \\
$0 \rightarrow 7$ & 3.59 & +0.00 & +0.00 & +0.00 & 0.00 \\
$0 \rightarrow 8$ & 3.70 & +0.00 & -0.09 & +0.00 & 0.00 \\
$0 \rightarrow 9$ & 3.80 & -0.25 & -0.00 & +0.00 & 0.01 \\
$0 \rightarrow 10$ & 3.83 & +0.00 & +0.14 & +0.00 & 0.00 \\
\hline
\end{tabular}

TABLE.S II. Transition properties of 5UT obtained by LRTDDFT calculation with PBE/6-31G(d). See the main text in this supplementary material for the details. 


\section{V: Geometry data of molecular systems}

We summarize the data of Cartesian coordinates in Angstrom for the molecular systems used in the present article. The information of atoms are also included below.

\section{NPTL-TCNE}

$\begin{array}{lrrr}\mathrm{C} & 1.824214 & 0.710474 & -1.999379 \\ \mathrm{C} & 1.824214 & -0.710473 & -1.999379 \\ \mathrm{C} & 0.628132 & -1.408746 & -1.999379 \\ \mathrm{C} & -0.619806 & -0.721697 & -1.999379 \\ \mathrm{C} & -0.619809 & 0.721696 & -1.999379 \\ \mathrm{C} & 0.628130 & 1.408742 & -1.999379 \\ \mathrm{C} & -1.867746 & 1.408746 & -1.999379 \\ \mathrm{C} & -3.063829 & 0.710474 & -1.999379 \\ \mathrm{C} & -3.063829 & -0.710473 & -1.999379 \\ \mathrm{C} & -1.867746 & -1.408742 & -1.999379 \\ \mathrm{H} & 2.775875 & 1.251891 & -1.999379 \\ \mathrm{H} & 2.775876 & -1.251890 & -1.999379 \\ \mathrm{H} & 0.624772 & -2.504937 & -1.999379 \\ \mathrm{H} & 0.624771 & 2.504934 & -1.999379 \\ \mathrm{H} & -1.864388 & 2.504937 & -1.999379 \\ \mathrm{H} & -4.015491 & 1.251889 & -1.999379 \\ \mathrm{H} & -4.015490 & -1.251891 & -1.999379 \\ \mathrm{H} & -1.864388 & -2.504934 & -1.999379 \\ \mathrm{~N} & 2.832029 & 2.070410 & 2.000621 \\ \mathrm{~N} & -1.591624 & 2.070452 & 2.000621 \\ \mathrm{C} & 1.839916 & 1.437817 & 2.000621 \\ \mathrm{C} & -0.599565 & 1.437776 & 2.000621 \\ \mathrm{C} & 0.620188 & 0.694253 & 2.000621 \\ \mathrm{C} & 0.620194 & -0.694285 & 2.000621 \\ \mathrm{C} & 1.839957 & -1.437800 & 2.000621 \\ \mathrm{C} & -0.599550 & -1.437827 & 2.000621 \\ \mathrm{~N} & 2.832015 & -2.070408 & 2.000621 \\ \mathrm{~N} & -1.591638 & -2.070398 & 2.000621\end{array}$

\begin{tabular}{lrrr} 
C & -9.102000 & 0.159612 & 0.000000 \\
S & -7.858600 & -1.051488 & 0.000000 \\
C & -6.587100 & 0.165912 & 0.000000 \\
C & -7.149500 & 1.439512 & 0.000000 \\
H & -9.185200 & 2.337012 & 0.000000 \\
H & -10.148900 & -0.142688 & 0.000000 \\
H & -6.546000 & 2.351012 & 0.000000 \\
$\mathrm{C}$ & -4.632800 & -1.495088 & 0.000000 \\
$\mathrm{C}$ & -5.196000 & -0.220788 & 0.000000 \\
$\mathrm{~S}$ & -3.927900 & 0.998512 & 0.000000 \\
$\mathrm{C}$ & -2.656500 & -0.219888 & 0.000000 \\
$\mathrm{C}$ & -3.219600 & -1.495488 & 0.000000 \\
$\mathrm{H}$ & -5.236800 & -2.406288 & 0.000000 \\
$\mathrm{H}$ & -2.615500 & -2.406588 & 0.000000 \\
$\mathrm{C}$ & -0.706100 & 1.441412 & 0.000000 \\
$\mathrm{C}$ & -1.270100 & 0.165512 & 0.000000 \\
$\mathrm{~S}$ & 0.000000 & -1.054088 & 0.000000 \\
$\mathrm{C}$ & 1.270100 & 0.165512 & 0.000000 \\
$\mathrm{C}$ & 0.706100 & 1.441412 & 0.000000 \\
$\mathrm{H}$ & -1.310300 & 2.352412 & 0.000000 \\
$\mathrm{H}$ & 1.310300 & 2.352412 & 0.000000 \\
$\mathrm{C}$ & 3.219600 & -1.495488 & 0.000000 \\
$\mathrm{C}$ & 2.656500 & -0.219888 & 0.000000 \\
$\mathrm{~S}$ & 3.927900 & 0.998512 & 0.000000 \\
$\mathrm{C}$ & 5.196000 & -0.220788 & 0.000000 \\
$\mathrm{C}$ & 4.632800 & -1.495088 & 0.000000 \\
$\mathrm{H}$ & 2.615500 & -2.406588 & 0.000000 \\
$\mathrm{H}$ & 5.236800 & -2.406288 & 0.000000 \\
$\mathrm{C}$ & 7.149500 & 1.439512 & 0.000000 \\
$\mathrm{C}$ & 6.587100 & 0.165912 & 0.000000 \\
$\mathrm{~S}$ & 7.858600 & -1.051488 & 0.000000 \\
$\mathrm{C}$ & 9.102000 & 0.159612 & 0.000000 \\
$\mathrm{C}$ & 8.572000 & 1.432812 & 0.000000 \\
$\mathrm{H}$ & 6.546000 & 2.351012 & 0.000000 \\
& 10.148900 & -0.142688 & 0.000000 \\
\hline & 9.185200 & 2.337012 & 0.000000
\end{tabular}

\section{2. $5 \mathrm{UT}$}

* takehiro.yonehara@riken.jp

† nakajima@riken.jp

1 G. R. Hutchison, M. A. Ratner, and T. J. Marks, J. Phys. Chem. A 106, 10596 (2002); Nitrogen-Containing Oligomers. In Electronic Materials: The Oligomer Approach; L. Groenendaal, E. W. Meijer, J. A. J. M. Vekemans, K. Müllen, G. Egner, Eds.; Wiley-VCH: Weinheim, 2007; pp 235-272

2 T. P. Kaloni, P. K. Giesbrecht, G. Schreckenbach, and M. S. Freund, Chem. Mater. 29, 10248 (2017).

3 T. Yanai, D. P. Tew, and N. C. Handy, Chem. Phys. Lett.
393, 51 (2004)

4 A. D. Becke, Phys Rev A 38, 3098 (1988); J.-W. Song, T. Hirosawa, T. Tsuneda, K. Hirao, J. Chem. Phys. 126, 154105 (2007); T. Tsuneda, K. Hirao, Chem. Phys. Lett. 268, 510 (1997)

5 S. Miertus, E. Scrocco, and J. Tomasi, Chem. Phys. 55, 117 (1981)

${ }^{6}$ M. W. Schmidt, K. K. Baldridge, J. A. Boatz, S. T. Elbert, M. S. Gordon, J. H. Jensen, S. Koseki, N. Matsunaga, K. A. Nguyen, and S. J. Su, J. Comput. Chem. 14, 1347 (1993) 